\title{
Stem cell transplantation: Current and prospective therapies to treat leukaemia
}

\author{
J ennifer R. Riggs, Stephen M. Wanta, Nikola Lekic, Matthew J. Craig, G. I an Gallicano \\ Department of Biochemistry and Molecular and Cell Biology, Georgetown University Medical Center, Washington, DC, USA \\ Correspondence: G. Ian Gallicano. Address: Department of Biochemistry and Molecular and Cell Biology, Georgetown \\ University Medical Center, Washington, DC, USA. Telephone: 1-202-687-0228. Fax: 1-202-687-1823. \\ Email: gig@georgetown.edu
}

Received: November 9, 2011

Accepted: November 29, 2011

Published: December 1, 2011

DOI : $10.5430 / j h m . v 1 n 1 p 24$

URL: http://dx.doi.org/10.5430/jhm.v1n1p24

\section{Abstract}

Stem cell therapies represent an important treatment option for leukemia patients, even with the expanding role of modern chemotherapies like tyrosine kinase inhibitors and monoclonal antibodies. Despite their strong curative potential, the use of hematopoietic stem cells carries a significant associated morbidity and mortality and is limited by the need to find an appropriately matched donor. While new stem cell sources like peripheral and umbilical cord blood (UCB) have increased the number of eligible patients, these therapies carry their own risks such as increased incidences of transplant related mortality with UCB. In this paper, we compare the effective outcomes of current stem cell therapies and their implications for use. Additionally, we also review the literature on emerging therapies like reduced intensity conditioning and donor lymphocyte infusion, and consider developing approaches like the use of mesenchymal stem cells and sex steroid ablation. Finally, we look at the potential applications of induced pluripotent stem cells and spermatogonial stem cells combined with gene correction as a future source of autologous stem cells for leukemia therapy.

\section{Key words}

Stem cells, Leukemia, Spermatogonial stem cells, Therapy

\section{I ntroduction}

Over the past twenty years, treatment options for leukemia have expanded, resulting in a steady decline in mortality. Leukemia is still the most common pediatric malignancy, representing thirty percent of all cancers in children under twenty years of age. While less prevalent in adults, overall survival for leukemia is lower compared to their pediatric counterparts. According to 2007 statistics, the overall incidence of leukemia is approximately eleven per 100,000 and the age-adjusted mortality rate is seven per 100,000 [1]. Significantly, age-dependent survival has increased from fourteen percent between 1980 and 1994 to twenty percent between 2000 and 2004 [2].

This improvement in survival for leukemia patients is in part due to the development of more effective chemotherapies, particularly regimens including tyrosine kinase inhibitors and monoclonal antibody therapies. These therapies have 
drastically affected the treatment of leukemia. Despite the effectiveness of non-transplant therapies, hematopoietic stem cell transplantation (HSCT) remains an important treatment option for many leukemic diseases [3, 4]. Because of the ability of quiescent leukemia stem cells to resist chemotherapy and radiation, the graft versus leukemia (GVL) effect of allogeneic HSCT represents one of the most powerful anti-leukemia treatments. In addition, HSCT still remains the only curative treatment for chronic myeloid leukemia (CML) [5].

There have been some studies showing improved disease-free survival (DFS) in patients undergoing HSCT compared to chemotherapy. However, the increased mortality related to HSCT may outweigh the efficacy in treating leukemia [5]. Reducing transplant-associated toxicities is a primary concern for increasing overall survival and decreasing morbidity and mortality associated with transplant therapy. Graft- versus-host disease (GVHD) is a major factor contributing to transplant-related mortality (TRM) in patients receiving HSCT [6]. However, GVHD is also associated with a GVL response. Therefore, it is important to balance any therapeutic control of GVHD with the increased risk of relapse [7]. Given the strong curative potential of HSCT, improving the survival of patients receiving this therapy has been attempted from multiple angles.

This multidimensional approach focuses on improving current sources of transplantation and incorporating novel therapies to mitigate TRM. In this paper, we review the relevant literature related to stem cell source and advancements and developing interventions in stem cell transplantations (SCT) for leukemia. We also consider the potential role of induced pluripotent stem cells (iPSCs) and spermatogonial stem cells (SSCs).

\subsection{Comparison of allogeneic stem cell sources}

\subsubsection{Bone marrow and peripheral blood progenitor cells}

Allogeneic SCT using peripheral blood progenitor cells (PBPCs) or bone marrow is one of the most effective stem cell therapies for improving DFS in certain populations of leukemia patients. In a meta-analysis of seven studies analyzing post-remission treatment of adult patients with acute lymphoblastic leukemia (ALL), Yanada et al. found that allogeneic HSCT was associated with increased overall survival compared to those who received chemotherapy, while autologous HSCT showed no increase in survival [8]. When high risk patients, determined by cytogenetic analysis, were the only group used in the analysis, overall survival was even greater indicating the importance of risk identification when determining treatment options for patients with ALL [8]. Another meta-analysis using a similar method of clinical trial selection but a different statistical model confirmed the findings of Yanada et al. and concluded that allogeneic transplantation has an acceptable cost effectiveness profile [9]. Koreth et al. have produced similar findings in patients with acute myeloid leukemia (AML), demonstrating improved outcomes for high risk patients undergoing HSCT compared to standard risk groups [10].

Results from the International ALL Trial showed that in both patients with Philadelphia chromosome-negative ALL and standard risk patients, post-remission allogeneic transplantation was able to provide increased overall survival in both groups and a decreased relapse rate in the Philadelphia chromosome-negative patients [11]. However, in contrast to other data, this trial saw no benefits to the high risk group with allogeneic HSCT, instead seeing higher TRM in high risk patients [11]. Further limiting the scope of HSCT, this trial demonstrated increased TRM in elderly patients [11]. These contradictory findings with regards to high risk patients highlight the need for careful balancing of the potential benefits of HSCT with the risk for TRM [10].

If problems arise finding an appropriate donor, chemotherapy or autologous HSCT can be substituted. In the International ALL Trial, autologous HSCT was compared to chemotherapy, and in all groups, patients receiving chemotherapy showed a significantly greater five-year survival rate [11]. However, patients receiving allogeneic HSCT showed greater anti-leukemia effects than patients in both the chemotherapy and autologous HSCT groups. In 2006, the American Society 
for Blood and Marrow Transplantation (ASBMT) recommended that allogeneic SCT should be used to treat adult ALL over autologous transplantation, but SCT yielded outcomes similar to chemotherapy and was therefore not recommended as the first line therapy. However, for high risk patients in their first complete remission (CR1), some data suggested an advantage for allogeneic SCT over chemotherapy [12]. In September 2011, the ASBMT updated their recommendations for ALL treatment. The new recommendations state that in the absence of a suitable allogeneic donor, autologous SCT may be an appropriate therapy compared to chemotherapy although the relapse rate is high, and that allogeneic SCT is an appropriate treatment for adult ALL in CR1 for all disease risk groups [13].

While these data confirm the usefulness of HSCT, further clarification on the applicability to specific risk groups is needed. Additional research has shown that for AML, bone marrow transplantation (BMT) has a better event free survival compared to chemotherapy [14]. In 2008, the ASBMT reported that for treatment of AML, there is no significant advantage of autologous SCT over chemotherapy, that HLA-matched donor SCT is recommended over autologous SCT and that there is a survival advantage for allogeneic HSCT versus chemotherapy for patients $>55$ with high risk cytogenetic [15].These data confirm the importance of HSCT and demonstrate the variability in response between different leukemia. However, further research comparing HSCT to newer chemotherapy agents is needed.

\subsubsection{Umbilical stem cells}

The use of umbilical cord blood (UCB) transplantation has increased the number of patients who are eligible for SCT. UCB offers many advantages compared to alternative sources of allogeneic stem cells including greater availability, an increase in eligible donors and decreased incidence of GVHD in comparison to similarly mismatched BMT and decreased risk of transmitting infections $[16,17,18]$. In HSCT, donors must be HLA matched, resulting in a small number of potential donors for a given patient. With UCB, multiple HLA mismatches can be tolerated, increasing the number of eligible donors [16, 19].

However, one significant disadvantage to using UCB is the decreased number of hematopoietic stem cells (HSCs) compared to bone marrow or peripheral blood which translates into a greater risk of graft failure which can potentially lead to increased mortality [16]. Additionally, UCB can result in delayed hematopoietic engraftment and increased hospital stays due to the need for blood and platelet transfusions [16]. Despite these drawbacks, UCB offers a much needed additional therapy option for patients who are unable to find a matched donor.

A study comparing the use of PBPC, BMT and UCB in 1525 adult patients diagnosed with either AML or ALL demonstrated that patients receiving UCB showed comparable relapse rates to PBPC or BMT, but TRM was significantly higher in the UCB group compared to matched PBPC and matched BMT (Figure 1) [20]. However, acute and chronic GVHD rates were lower in the UCB groups [20]. Additional studies have focused on AML and ALL separately and have shown that in AML, UCB mismatched at no more than two antigens is associated with decreased overall survival and decreased DFS compared to BMT [21]. Although the relapse rate did not differ among the treatment groups, TRM was significantly higher in the UCB group [21]. The data were more encouraging in ALL as there were no significant differences between UCB and BMT for relapse, TRM, overall survival, and DFS [21].

These data suggest that while UCB has an important role in leukemia treatment, it should currently only be considered in cases where a matched HLA donor cannot be found. Increased TRM associated with slow rates of hematopoietic and functional T cell recovery have limited the use of UCB in adults, and novel strategies need to be explored and developed to improve UCB patient outcomes and expand the role of HSCT in treating leukemia. 


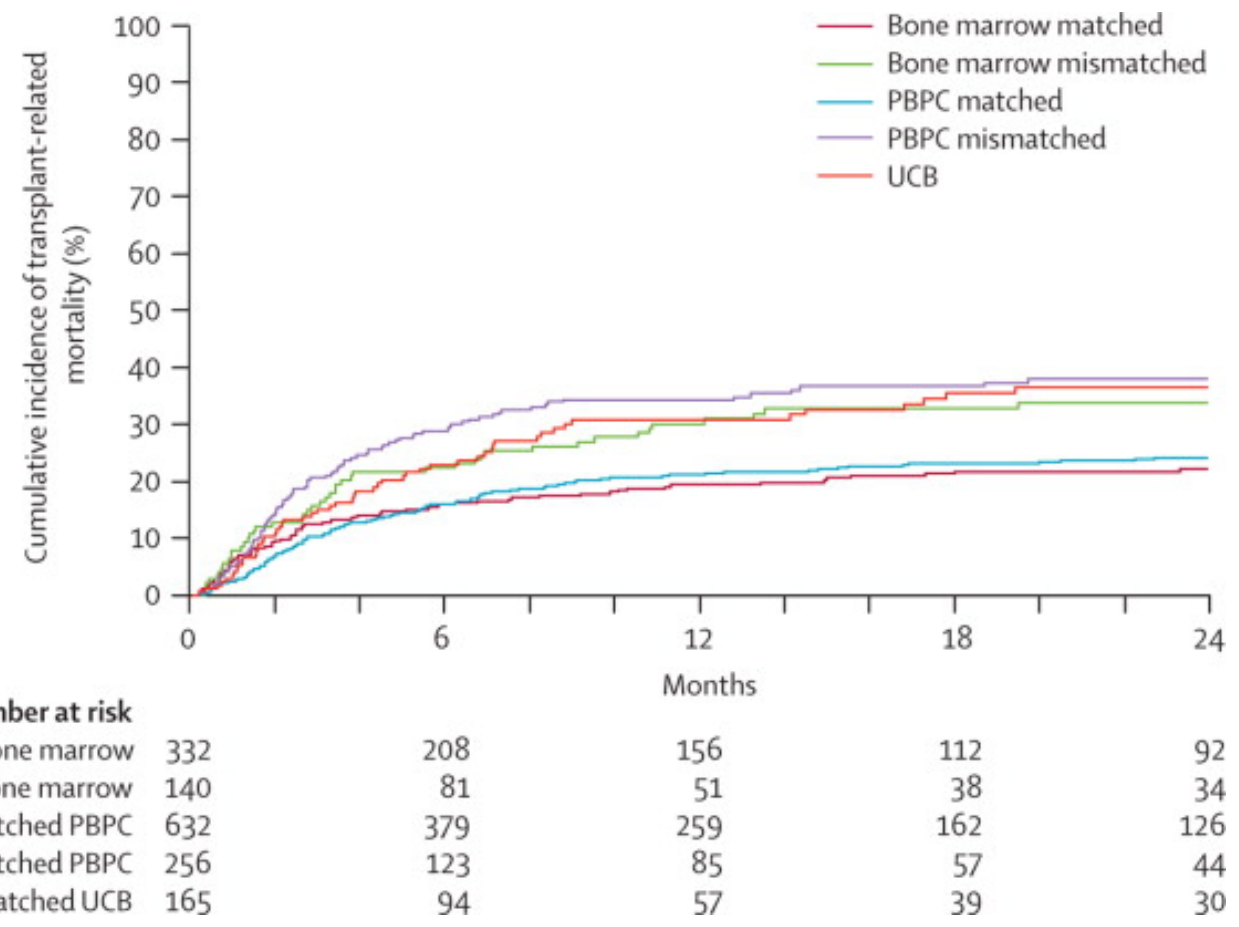

Figure 1. The probabilities of transplant-related mortality by hematopoietic stem-cell source and HLA matching The 2-year adjusted probability of transplant-related mortality after transplantation of 4-6/6 HLA matched umbilical-cord blood (UCB), 8/8 HLA-matched peripheral blood progenitor cells (PBPCs), 8/8 HLA-matched bone marrow, 7/8 HLA-matched PBPCs, and 7/8 HLA-matched bone marrow was 37\%, 24\%, 22\%, 38\% and 34\%, respectively (20).

\section{Novel therapies}

\subsection{Pretransplantation therapies}

Reduced-intensity conditioning (RIC) is a therapy in which the patient is given a lower dose of chemotherapy or chemoradiation to prevent the immune system from being completely compromised. RIC has been associated with decreased TRM while maintaining a sufficient GVL effect [22-25]. The reduction in TRM seen with RIC is likely due to decreased risk of infection and/or less severe damage of host defense mechanisms. However, studies have shown increased relapse risk with RIC compared to standard myeloablative conditioning (MAC) [26]. An RIC regimen is typically prescribed instead of MAC to patients who would benefit from the decrease in immunosuppression due to advanced age or complicating conditions. One limitation of RIC and MAC comparison studies is that RIC recipients tend to be older. However, the efficacy of RIC has been found to be independent of age and overall survival was comparable between RIC and MAC regimens [22, 26]. Additionally, several studies have shown that patients pretreated with RIC had decreased incidence of acute grade II-IV GVHD [22, 27]. Further investigation should be carried out to determine the specific mechanism of RIC-associated TRM reduction and the relationship between TRM and the increased relapse risk observed with RIC. The relationship between RIC and GVHD should also be explored.

Pretreatment of donors with immunomodulators prior to allogeneic stem cell transplantation is another novel strategy being tested in mouse models. Results have shown that pretreatment prevents development of GVHD while concomitantly inducing long-lasting GVL effects [28, 29]. This is a promising new treatment that will hopefully encourage further attempts to develop an effective cell therapy with immunomodulators for clinical application. 


\subsection{Post transplantation therapies}

Donor lymphocyte infusion (DLI) is the standard form of immunotherapy given to post transplant leukemia patients to prevent and treat relapse. Donor lymphocyte cells react against leukemia cells producing a GVL effect and also help facilitate host immune reconstitution. Yet in a retrospective analysis, sixty percent of patients developed chronic GVHD after DLI treatment, but remission was rarely observed in patients without GVHD after DLI [30]. DLI is typically administered several weeks after stem cell transplantation, so it is probable that donor reconstitution of the host immune system helps mitigate graft-versus-host (GVH) effects. The correlation between increased GVHD and GVL reactivity in DLI-treated patients exists because regulatory $\mathrm{T}$ cells and host antigen-presenting cells (APCs) are involved in both GVHD and GVL reactivity. Xia et al. found that donor Thy1+ regulatory cells suppress GVH effects after DLI treatment, and that elimination of donor Thy1+ cells resulted in lethal acute GVHD and loss of GVL effects [30]. In murine models, the suppression of GVHD by Thy1+ cells was not required in the long term because of the turnover of APCs from host to donor, preventing the activation of alloreactive T cells; therefore, the presence of host APCs induces GVHD after DLI treatment in mice. However, in murine models, host APCs are required for initiating GVL effects after DLI therapy [30, 31]. More research is required to understand the immunological mechanisms involved in GVHD and GVL reactivity after DLI treatment in order to enhance GVL effects and to optimize clinical usage for DLI-based immunotherapy.

Translational studies have also shown that DLI is able to increase GVL response without GVHD in mixed chimeric mice. Data have shown that a state of mixed chimerism is superior because it preserves the host APCs essential for initiating GVL [30, 31]. Host APCs also play a role in GVH reactions, but GVH reactions in mixed chimeric mice after DLI are confined to hematopoietic tissues, preventing diffuse GVHD [30]. While this is an exciting new development, its effect has only been shown in mouse models. Further investigation needs to be carried out to elucidate the mechanisms of chimerism in both mice and humans and to determine its immunological effects.

Another novel posttransplant therapy being explored in murine models is sex steroid ablation using leuprolide acetate, a luteinizing hormone-releasing hormone agonist (LHRHa). Posttransplant immunodeficiency, particularly the lack of T cell reconstitution, is a major complication of allogeneic SCT and a major cause of TRM and relapse. Previous research has shown that androgen receptors are expressed on adult lymphoid precursors and CD34 + human cord blood cells. Goldberg et al. have shown that posttransplant treatment with LHRHa increases the number of lymphoid and myeloid progenitor cells in the bone marrow and developing thymocytes in the thymus without exacerbating GVHD and maintaining GVL effects [32]. This novel approach represents a potentially useful clinical tool to reverse thymic atrophy and enhance immunity following immunosuppression. While LHRHa therapy for leukemia has only been tested in mice, LHRHa has a strong safety profile, demonstrated by its clinical use in patients with precocious puberty and breast and prostate cancer [32-36].

\subsubsection{Mesenchymal stem cells}

Mesenchymal stem cells (MSCs) are multipotent bone marrow cells capable of self-renewal and multidifferentiation into tissues of mesenchymal origin [37, 38]. MSCs also modulate the immune response in vitro and in vivo and have been shown to promote engraftment of HSCs in animal models [37]. In a series of several studies, Zhao et al. found that MSCs from bone marrow of patients with ALL were similar to MSCs derived from normal adult bone marrow in phenotype, morphology and differentiation capacity [38]. ALL-derived MSCs also produced hematopoietic cytokines and supported hematopoiesis in long-term culture. Similar results were obtained comparing MSCs derived from the bone marrow of patients with CML with MSCs derived from normal adult bone marrow. However, MSCs derived from patients with AML showed abnormal biological properties, limited proliferation capacity, and impaired differentiation and hematopoiesis support ability [38]. While further research should be done to understand the differences among cells derived from different disease states, MSCs from the bone marrow of ALL and CML patients could be used to provide an ex vivo hematopoietic support environment during progenitor cell expansion. Additionally, because MSCs mediate systemic immunosuppression in vivo, co-transplantation of MSCs derived from ALL and CML patients could promote engraftment of HSCs. 
In another study, HLA-identical MSCs expanded ex vivo were infused into fifty-five patients with steroid-resistant, acute GVHD to promote hematopoietic recovery after autologous and allogeneic HSCT. Thirty-nine patients responded to treatment, and survival was higher and TRM significantly lower in patients exhibiting a complete response compared to those with partial or no response. Two years later, no acute or long-term adverse effects had been reported [37]. This study suggests that MSCs may be an effective treatment for patients with steroid-resistant, acute GVHD who do not respond to other immunosuppressive therapies. However, additional studies are needed to determine the best dose of MSCs and the number of infusions needed to increase patient outcomes. Tracking studies should also be performed to determine how long MSCs survive post-injection and how MSCs target tissues.

MSCs represent a promising new therapeutic approach. MSCs can be used to promote ex vivo expansion of progenitor cells or in conjunction with current stem cell therapies to promote engraftment in vivo. Several studies have shown that MSCs are an effective treatment against steroid-refractory acute GVHD [37, 39, 40], but additional studies should be performed to understand the mechanisms of suppression of GVHD and to determine if MSCs affect GVL reactivity. Randomized studies should be done to determine if the immunosuppression caused by MSCs in vivo increases TRM related to infection and relapse risk. If these preliminary data are reinforced by further studies, the use of MSCs as an autologous stem cell therapy should be considered a primary research objective.

\subsubsection{Autologous transplantation and new stem cell sources}

Allogeneic HSCT currently offers the best curative option for leukemia patients, but there is still a role for autologous transplants in patients who cannot tolerate GVHD. While autologous HSCT does not offer the same GVL effects as allogeneic HSCT, the lack of GVHD reduces TRM. In autologous HSCT, there is often difficulty in harvesting an uncontaminated sample from a patient, leading to a shorter period of DFS. The prospect of generating HSCs using iPSCs or SSCs offers a new source of uncontaminated cells without the ethical complications of embryonic stem cells.

\subsubsection{I nduced pluripotent stem cells}

iPSCs are generated from differentiated adult cells that have been reprogrammed to become pluripotent stem cells with embryonic cell-like properties that then have the potential to undergo gene therapy and subsequent differentiation to various cell lineages - hematopoietic stem cells in the case of leukemia. Takahashi et al. reprogrammed adult human fibroblasts into pluripotent stem cells by retroviral transduction using only four factors: Oct-4, SOX2, KLF4, and c-Myc [41]. Furthermore, the iPSCs generated show similar gene expression to embryonic stem cells and are capable of teratoma formation [41]. However, some of the known limitations of traditional iPSC development via retrovirus transduction include the introduction of potentially deleterious effects such as genomic instability, tumor formation, and the lengthy time requirements needed to obtain these cells [41-50].

Therefore, more recent studies have avoided reprogramming adult cells with retroviral vectors [51, 52]. Hu et al. successfully reprogrammed bone marrow mononuclear cells from both normal patients and patients with CML using the same reprogramming factors used by Takahashi et al. but with non-integrating Epstein Bar Virus episomal vectors - a double-stranded DNA, non-retroviral mechanism [51]. Positive assays for teratoma formation and gene expression corroborated the group's claim of pluripotency [51]. After reprogramming the cells to pluripotency, Hu et al. co-cultured the iPSCs on OP9 stromal cells containing hematopoietic inductive factors. The cultured bone marrow-derived iPSCs produced a modest amount of CD43+, a known hematopoietic cell marker, whereas studies by Choi et al. using human iPSCs generated from adult fibroblast cells produced significantly more CD43+ hematopoietic progenitor cells when co-cultured with OP9 cells [51,53]. The discrepancy in hematopoietic progenitor efficiency is likely due to the resistance of iPSCs to differentiate back to the parental myeloid cells whose cell type predominates in the cultures of iPSC hematopoietic differentiation [51]. Therefore, fibroblast-derived iPSCs are likely the better option over bone marrow-derived iPSCs for differentiation into hematopoietic progenitor cells due to their higher efficiency. Additionally, the reprogrammed CML bone marrow cells displaying embryonic stem cell-like characteristics can be used in future studies of molecular mechanisms of disease progression and drug resistance in CML patients [51]. Although Hu et al. 
claim a non-integrative Epstein Bar Virus iPSC method, investigators are apprehensive about using stem cells reprogrammed with viral vectors in clinical trials, primarily due to genomic instability [52].

Investigation into protein reprogramming has achieved moderate success [52]. Kim et al. reprogrammed human fibroblast cells into iPSCs using the four reprogramming factors Oct-4, SOX2, KLF4, and c-Myc fused to the amino acid arginine in the ninth position of a cell-penetrating peptide [52]. Western blot analysis confirmed stability of the reprogramming factors in the iPSCs [52]. Furthermore, differentiation into various cell types from all three germ layers, confirmed by immunocytochemistry and teratoma formation, demonstrates the pluripotency of those iPSCs [52]. Although the concept of non-viral reprogramming of differentiated cells to a pluripotent state is encouraging, there are still many limitations including reprograming time and low efficiency [52]. Therefore, investigators are searching for pluripotent stem cell sources without the need for reprogramming protein integration.

\subsubsection{Spermatogonial stem cells}

Another source of pluripotent stem cells are SSCs derived from testes. Golestaneh et al. isolated human germ cells from donor testes and dedifferentiated the cells to a pluripotent stem cell state by growing them on an embryonic stem cell-like knockout culture [54]. Subsequently, they demonstrated the pluripotency of these dedifferentiated SSCs through teratoma formation and differentiation to all three germ layers: endodermal islet-like cells, mesodermal cardiac cells, and an ectodermal neural lineage [54]. Further investigations by Yoshimoto et al. demonstrated that SSCs incubated on an OP9 co-culture differentiated into hematopoietic progenitor cells of $\mathrm{T}, \mathrm{B}$, and erythromyeloid cell lineages in a murine model [55]. Additionally, successful transplantation by direct injection of these hematopoietic stem cells into the bone marrow of non-obese diabetic/severe combined immunodeficient mice was achieved even four months after engraftment [55].

The primary advantage of using SSC over iPSC methods is removing the need to introduce the four reprogramming factors imperative for iPSC reprogramming, thus avoiding complications with dedifferentiation. Furthermore, research demonstrating successful dedifferentiation to an embryonic stem cell-like state of human SSCs in addition to the advancements in developing hematopoietic progenitor cell lineages and bone marrow engraftment in the murine SSC model has led investigators closer to finding an autologous treatment for male patients suffering from leukemia [54, 55]. The future of this concept must overcome one final obstacle - an oncogenic genome.

\subsection{Gene therapy}

Leukemia is a complex group of diseases with a poorly understood pathogenesis, partially attributed to various unknown genetic interactions. Therefore, gene therapy for such a disease is currently out of reach. Nevertheless, when the intricacies of leukemia are one day elucidated, autologous stem cell transplantation in conjunction with gene therapy may play a major role in a cure. Significant progress in this area has been made on more simple blood-borne diseases [56].

Recently, investigators have successfully used in vitro methods to correct for a mutated gene in bone marrow cells of patients suffering from the blood disorder paroxysmal nocturnal hemoglobinuria by using both human iPSCs and human embryonic stem cells [56]. These investigations by Zou et al. are significant because they are the first to demonstrate modification of human iPSCs with homologous recombination mediated by zinc finger nuclease [56]. Just like with iPSC reprogramming, non-viral gene therapies are preferred in order to avoid genomic instability, which the zinc finger nucleases used by Zou et al. offer [56]. Future research improving upon non-viral gene therapies such as zinc finger nucleases in conjunction with multi-gene manipulation may lead investigators closer to finding an autologous therapy for leukemia patients.

\section{Conclusions}

Transplantation with HSCs has improved greatly over the past decade, changing its role from a last option therapy to a frontline treatment during first or second remission, especially in patients with matched donors. A primary goal for HSCT 
is improving the number of potential donors. To this end, it is important to ameliorate outcomes for current sources for HSCs. Currently, HLA matched allogeneic BMT represents the best curative option for many leukemia patients. However, matched donors exist for less than thirty percent of patients seeking transplant. UCB is often the best option for patients with no matched donors, since GVHD is less likely to occur relative to unmatched BMT. The increasing use of UCB has expanded the number of patients able to receive stem cell transplantation, but adds complicating factors of its own, such as increased TRM.

In addition to developing new sources of HSCs, there is also a growing body of research supporting new or modified conjunctive therapies. A particular area of focus has been on conditioning regimens, which have traditionally made HSCT contraindicated for many older leukemia patients. RIC has allowed for elderly patients or patients with coexisting illnesses to receive HSCT [22]. While the occurrence of relapse is higher in patients receiving RIC, the improvements in GVHD are notable and the mechanism behind this deserves further exploration. On the post transplantation side, DLI has shown promising results in reducing GVHD while maintaining a strong GVL response [30].

Given the development of many novel approaches to stem cell therapies for leukemia, the curative role for HSCT is expanding. The experiments in murine models using sex steroid ablation with LHRHa could help reduce TRM in patients undergoing UCB transplantation as well as other forms of HSCT [32]. Also, the results from studies incorporating MSCs into posttransplantion therapy show an effective mechanism for treating GVHD, particularly in steroid-resistant patients. However, more research is needed to determine the effects on GVL and the applicability to other patient populations.

In addition to enhancing current stem cell sources, the possibility of generating HSCs using iPSCs or SSCs with genetic modifications to correct for oncogenic properties may impact the way leukemia is treated in the future. Autologous transplantation is generally less effective than allogeneic transplantation due to the lack of GVL effects and the contamination of autologous sources, as well as the transmission of any oncogenic properties that may be present in the genome [5]. However, iPSCs and SSCs represent an uncontaminated autologous source that has the added potential benefit of genome modifications to eradicate existing oncogenes. Thus, these therapies may become a preferred treatment option for patients who cannot tolerate a GVH response, such as the elderly. Animal studies are needed to evaluate the true curative potential of this therapy in leukemia.

Given these increasing therapeutic options, it is important to consider differences in the various patient populations when evaluating stem cell treatment. Factors such as age, type of leukemia and severity of illness greatly impact the indications for HSCT. For example, HSCT is frequently used in first line treatment of pediatric CML, while adults traditionally receive a tyrosine kinase inhibitor [57]. In the case of pre and post transplantation therapies, RIC may be more beneficial for older patients who are more susceptible to TRM. As new therapies become available, physicians must consider the effect on relapse as well as overall survival, applied to specific patient populations.

Overall, the focus on expanding stem cell sources and improving transplant related complications has opened stem cell therapy to an increasing number of leukemia patients. Morbidity and mortality associated with HSCT still represents a powerful obstacle in their use as a front line therapy, especially for elderly patients. However, current and prospective conjunctive therapies are promising in their ability to mitigate TRM and improve outcomes.

\section{Conflict of interest}

The authors declare that they have no Conflict of interest.

\section{References}

[1] Surveillance, Epidemiology, and End Results (SEER) Program. www.seer.cancer.gov (6 March 2011, date last accessed). 
[2] Pulte D, Gondos A, Brenner H. Improvement in survival in younger patients with acute lymphoblastic leukemia from the 1980s to the early 21st century. Blood. 2009;113:1408-11.

[3] Ohno R. Treatment of adult patients with Philadelphia chromosome-positive acute lymphoblastic leukemia. Curr Oncol Rep. 2008;10:379-87. PMid: 3987412.

[4] Fielding AK, Rowe JM, Richards SM, Buck G, Moorman AV, Durrant IJ, et al. Prospective outcome data on 267 unselected adult patients with Philadelphia chromosome-positive acute lymphoblastic leukemia confirms superiority of allogeneic transplantation over chemotherapy in the pre-imatinib era: results from the International ALL Trial. Blood. 2009;19:4489-96.

[5] Copelan EA. Hematopoietic Stem-Cell Transplantation. N Engl J Med. 2006;354:1813-26.

[6] Fagioli F, Zecca M, Rognoni C, Lanino E, Balduzzi A, Berger M, et al. Allogeneic Hematopoietic Stem Cell Transplantation for Philadelphia Positive Acute Lymphoblastic Leukemia in Children and Adolescents: a Retrospective Multicenter Study of the Italian Association of Pediatric Hematology and Oncology (AIEOP). Biol Blood Marrow Transplant. 2011[Epub ahead of print].

[7] Hari P, Logan B, Drobyski WR. Temporal discordance between graft-versus-leukemia and graft-versus-host responses: a strategy for the separation of graft-versus-leukemia/graft-versus-host reactivity? Biol Blood Marrow Transplant. 2004;10:743-47. PMid: 19254107.

[8] Yanada M, Matsuo K, Suzuki T, Naoe T. Allogeneic hematopoietic stem cell transplantation as part of postremission therapy improves survival for adult patients with high-risk acute lymphoblastic leukemia: a metaanalysis. Cancer. 2006;106:2657-63.

[9] Bartolozzi B, Bosi A, Orsi C. Allogeneic hematopoietic stem cell transplantation as part of postremission therapy improves survival for adult patients with high-risk acute lymphoblastic leukemia: a meta-analysis. Cancer. 2007;109: 343-44.

[10] Koreth J, Schlenk R, Kopecky KJ, Honda S, Sierra J, Djulbegovic BJ, et al. Allogeneic stem cell transplantation for acute myeloid leukemia in first complete remission: systematic review and meta-analysis of prospective clinical trials. JAMA. 2009;301:2349-61. PMid: 18410208.

[11] Goldstone AH, Richards SM, Lazarus HM, Tallman MS, Buck G, Fielding AK, et al. In adults with standard-risk acute lymphoblastic leukemia, the greatest benefit is achieved from a matched sibling allogeneic transplantation in first complete remission, and an autologous transplantation is less effective than conventional consolidation/maintenance chemotherapy in all patients: final results of the International ALL Trial (MRC UKALL XII/ECOG E2993). Blood. 2008;111:1827-33.

[12] Hahn T, Wall D, Camitta B, et al. The role of cytotoxic therapy with hematopoietic stem cell transplantation in the therapy of acute lymphoblastic leukemia in adults: An evidence-based review. Biol Blood Marrow Transplant. 2006;12:1-30.

[13] ASBMT Position Statement. The Role of Cytotoxic Therapy with Hematopoietic Stem Cell Transplantation, in the Treatment of Adult Acute Lymphoblastic Leukemia: Update of the 2006 Evidence-Based Review. Biol Blood Marrow Transplant. [In press].

[14] Levi I, Grotto I, Yerushalmi R, Ben-Bassat I, Shpilberg O. Meta-analysis of autologous bone marrow transplantation versus chemotherapy in adult patients with acute myeloid leukemia in first remission. Leuk Res. 2004;28:605-12. PMid: 10740682.

[15] Oliansky DM, Appelbaum F, Cassileth PA, Keating A, Kerr J, Nieto Y, et al. The role of cytotoxic therapy with hematopoietic stem cell transplantation in the therapy of acute myelogenous leukemia in adults: an evidence-based review. Biol Blood Marrow Transplant. 2008;14:137-80.

[16] Rocha V, Gluckman E. Clinical use of umbilical cord blood hematopoietic stem cells. Biol Blood Marrow Transplant. 2006;12:34-41.

[17] Sanz J, Sanz GF. Umbilical cord blood transplantation from unrelated donors in adult patients with chronic myeloid leukaemia. Best Pract Res Clin Haematol. 2010.23:217-22.

[18] Wagner JE, Gluckman E. Umbilical cord blood transplantation: the first 20 years. Semin Hematol. 2010;47:3-12.

[19] Delaney M, Ballen KK. The role of HLA in umbilical cord transplantation. Best Pract Res Clin Haematol. 2010;23:179-87.

[20] Eapen M, Rocha V, Sanz G, Scaradavou A, Zhang MJ, Arcese W, et al. Effect of graft source on unrelated donor haemopoietic stem-cell transplantation in adults with acute leukaemia: a retrospective analysis. Lancet Oncol. 2010;11:653-60.

[21] Atsuta Y, Suzuki R, Nagamura-Inoue T, Taniguchi S, Takahashi S, Kai S, et al. Disease-specific analyses of unrelated cord blood transplantation compared with unrelated bone marrow transplantation in adult patients with acute leukemia. Blood. 2009;113:1631-38.

[22] Marks DI, Wang T, Pérez WS, Antin JH, Copelan E, Gale RP, et al. The outcome of full-intensity and reduced-intensity conditioning matched sibling or unrelated donor transplantation in adults with Philadelphia chromosome-negative acute lymphoblastic leukemia in first and second complete remission. Blood. 2010;116:366-74.

[23] Bachanova V, Verneris MR, DeFor T, Brunstein CG, Weisdorf DJ. Prolonged survival in adults with acute lymphoblastic leukemia after reduced-intensity conditioning with cord blood or sibling donor transplantation. Blood. 2009;113:2902-05.

[24] Dreger P, Brand R, Hansz J, Milligan D, Corradini P, Finke J, et al. Treatment-related mortality and graft-versus-leukemia activity after allogeneic stem cell transplantation for chronic lymphocytic leukemia using intensity-reduced conditioning. Leukemia.

2003;17:841-48. 
[25] Schetelig J, Thiede C, Bornhauser M, Schwerdtfeger R, Kiehl M, Beyer J, et al. Evidence of a graft-versus-leukemia effect in chronic lymphocytic leukemia after reduced-intensity conditioning and allogeneic stem-cell transplantation: the Cooperative German Transplant Study Group. J Clin Oncol. 2003;21:2747-53.

[26] Mohty M, Labopin M, Volin L, Gratwohl A, Socié G, Esteve J, et al. Reduced-intensity versus conventional myeloablative conditioning allogeneic stem cell transplantation for patients with acute lymphoblastic leukemia: a retrospective study from the European Group for Blood and Marrow Transplantation. Blood. 2010;116:4439-43.

[27] Pérez-Simón JA, Díez-Campelo M, Martino R, Brunet S, Urbano A, Caballero MD, et al. Influence of the intensity of the conditioning regimen on the characteristics of acute and chronic graft-versus-host disease after allogeneic transplantation. Br $\mathrm{J}$ Haematol. 2005;130:394-403.

[28] Morecki S, Yacovlev E, Gelfand Y, Shabat Y, Slavin S. Induction of graft-versus-leukemia (GVL) effect without graft-versus-host disease (GVHD) by pretransplant donor treatment with immunomodulators. Biol Blood Marrow Transplant. 2009;15:406-15.

[29] Morecki S, Yacovlev E, Gefland Y, Eizik O, Slavin S. Pretransplant treatment of donors with immunomodulators to control graft-versus-host disease (GVHD) in transplant recipients. Exp Hematol. 2007;35:748-56.

[30] Xia G, Truitt RL, Johnson BD. Graft-versus-leukemia and graft-versus-host reactions after donor lymphocyte infusion are initiated by host-type antigen-presenting cells and regulated by regulatory T cells in early long-term chimeras. Biol Blood Marrow Transplant. 2006;12:397-407.

[31] Mapara MY, Kim YM, Marx J, Sykes M. Donor lymphocyte infusion-mediated graft-versus-leukemia effects in mixed chimeras established with nonmyeloablative conditioning regimen: extinction of graft-versus-leukemia effects after conversion to full donor chimerism. Transplantation. 2003;76:297-305.

[32] Goldberg GL, King CG, Nejat RA, Suh DY, Smith OM, Bretz JC, et al. Luteinizing hormone-releasing hormone enhances T cell recovery following allogeneic bone marrow transplantation. J Immunol. 2009;182:5846-54.

[33] Crowley WR Jr, Comite F, Vale W, Rivier J, Loriaux DL, Cutler GB Jr. Therapeutic use of pituitary desensitization with a long-acting lhrh agonist: a potential new treatment for idiopathic precocious puberty. J Clin Endocrinol Metab. 1981;52:370-72.

[34] Klein KO, Barnes KM, Jones JV, Feuillan PP, Cutler GB Jr. Increased final height in preococious puberty after long-term treatment with LHRH agonists: the National Institutes of Health experience. J Clin Endocrinol Metab. 2001;86:4711-16.

[35] Miller WR, Scott WN, Morris R, Fraser HM, Sharpe RM. Growth of human breast cancer cells inhibited by a luteinizing hormone-releasing hormone agonist. Nature. 1985;313:231-33.

[36] Bolla M, Collette L, Blank L, Warde P, Dubois JB, Mirimanoff RO, et al. Long-term results with immediate androgen suppression and external irradiation in patients with locally advanced prostate cancer (an EORTC study): a phase III randomised trial. Lancet. 2002;360:103-08.

[37] LeBlanc K, Frassoni F, Ball L, Locatelli F, Roelofs H, Lewis I, et al. Mesenchymal stem cells for treatment of steroid-resistant, severe, acute graft-versus-host disease: a phase II study. Lancet. 2008;371:1579-86.

[38] Zhao ZG, Liang Y, Li K, Li WM, Li QB, Chen ZC, et al. Phenotypic and functional comparison of mesenchymal stem cells derived from the bone marrow of normal adults and patients with hematologic malignant diseases. Stem Cells Dev. 2007;16:637-48.

[39] Ringdén O, Uzunel M, Rasmusson I, Remberger M, Sundberg B, Lönnies H, et al. Mesenchymal stem cells for treatment of therapy-resistant graft-versus-host disease. Transplantation. 2006;81:1390-97.

[40] Fang B, Song Y, Liao L, Zhang Y, Zhao RC. Favorable response to human adipose tissue-derived mesenchymal stem cells in steroid-refractory acute graft-versus-host disease. Transplant Proc. 2007;39:3358-62.

[41] Takahashi K, Tanabe K, Ohnuki M, Narita M, Ichisaka T, Tomoda K, et al. Induction of pluripotent stem cells from adult human fibroblasts by defined factors. Cell. 2007;131:861-72.

[42] Cho HJ, Lee CS, Kwon YW, Paek JS, Lee SH, Hur J, et al. Induction of pluripotent stem cells from adult somatic cells by protein-based reprogramming without genetic manipulation. Blood. 2010;116:386-95.

[43] Kaji K, Norrby K, Paca A, Mileikovsky M, Mohseni P, Woltjen K. Virus-free induction of pluripotency and subsequent excision of reprogramming factors. Nature. 2009;459:771-75.

[44] Okita K, Hong H, Takahashi K, Tamanaka S. Generation of mouse-induced pluripotent stem cells with plasmid vectors. Nat Protoc. 2010;5:418-28.

[45] Rhee YH, Ko JY, Chang MY, Yi SH, Kim D, Kim CH, et al. Protein-based human iPS cells efficiently generate functional dopamine neurons and can treat a rat model of Parkinson disease. J Clin Invest. 2011;121:2326-35.

[46] Tavernier G, Wolfrum K, Demeester J, De Smedt SC, Adjaye J, Rejman J. Activation of pluripotency-associated genes in mouse embryonic fibroblasts by non-viral transfection with in vitro-derived mRNAs encoding Oct4, Sox2, Klf4 and cMyc. Biomaterials. 2011[Epub ahead of print] 
[47] Tsukiyama T, Asano R, Kawaguchi T, Kim N, Yamada M, Minami N, et al. Simple and efficient method for generation of induced pluripotent stem cells using piggyBac transposition of doxycycline-inducible factors and an EOS reporter system. Genes Cells. 2011;16:815-25.

[48] Karow M, Chavez CL, Farruggio AP, Geisinger JM, Keravala A, Jung WE, et al. Site-specific recombinase strategy to create induced pluripotent stem cells efficiently with plasmid DNA. Stem Cells. 2011;29:1696-704.

[49] Woltjen K, Michael IP, Mohseni P, Desai R, Mileikovsky M, Hamalainen R, et al. piggyBac transposition reprograms fibroblasts to induced pluripotent stem cells. Nature. 2009;458:766-70.

[50] Yu J, Hu K, Smuga-Otto K, Tian S, Stewart R, Slukvin II, et al. Human induced pluripotent stem cells free of vector and transgene sequences. Science. 2009;324:797-801.

[51] Hu K, Yu J, Suknuntha K, Tian S, Montgomery K, Choi KD, et al. Efficient generation of transgene-free induced pluripotent stem cells from normal and neoplastic bone marrow and cord blood mononuclear cells. Blood. 2011;117:109-19.

[52] Kim D, Kim CH, Moon JI, Chung YG, Chang MY, Han BS, et al. Generation of human induced pluripotent stem cells by direct delivery of reprogramming proteins. Cell Stem Cell. 2009;4:472-76.

[53] Choi KD, Yu J, Smuga-Otto K, Salvagiotto G, Rehrauer W, Vodyanik M, et al. Hematopoietic and endothelial differentiation of human induced pluripotent stem cells. Stem Cells. 2009;27:559-67.

[54] Golestaneh N, Kokkinaki M, Pant D, Jiang J, DeStefano D, Fernandez-Bueno C, et al. Pluripotent stem cells derived from adult human testes. Stem Cells Dev. 2009;18:1115-26.

[55] Yoshimoto M, Heike T, Chang H, Kanatsu-Shinohara M, Baba S, Varnau JT, et al. Bone marrow engraftment but limited expansion of hematopoietic cells from multipotent germline stem cells derived from neonatal mouse testis. Exp Hematol. 2009;37:1400-10.

[56] Zou J, Maeder ML, Mali P, Pruett-Miller SM, Thibodeau-Beganny S, Chou BK, et al. Gene targeting of a disease-related gene in human induced pluripotent stem and embryonic stem cells. Cell Stem Cell. 2009;5:97-110.

[57] Suttorp M, Yaniv I, Schultz KR. Controversies in the treatment of CML in children and adolescents: TKIs versus BMT? Biol Blood Marrow Transplant Suppl. 2011;17:S115-S122 